\title{
Another Generalized Transmuted Family of Distributions: Properties and Applications
}

\author{
Faton Merovci \\ University of Mitrovica
}

\author{
Morad Alizadeh \\ Persian Gulf University
}

\author{
G. G. Hamedani \\ Marquette University
}

\begin{abstract}
We introduce and study general mathematical properties of a new generator of continuous distributions with two extra parameters called the Another generalized transmuted family of distributions. We present some special models. We investigate the asymptotes and shapes. The new density function can be expressed as a linear combination of exponentiated densities based on the same baseline distribution. We obtain explicit expressions for the ordinary and incomplete moments and generating functions, Bonferroni and Lorenz curves, asymptotic distribution of the extreme values, Shannon and Rényi entropies and order statistics, which hold for any baseline model, certain characterisations are presented. Further, we introduce a bivariate extensions of the new family. We discuss the different method of estimation of the model parameters and illustrate the potentiality of the family by means of two applications to real data. A brief simulation for evaluating Maximum likelihood estimator is done.
\end{abstract}

Keywords: transmuted distribution, generated family, maximum likelihood, moment, order statistic, quantile function, Rényi entropy, characterizations..

\section{Introduction}

Numerous classical distributions have been extensively used over the past decades for modeling data in several areas such as engineering, actuarial, environmental and medical sciences, biological studies, demography, economics, finance and insurance. However, in many applied areas such as lifetime analysis, finance and insurance, there is a clear need for extended forms of these distributions. For that reason, several methods for generating new families of distributions have been studied. Some attempts have been made to define new families of probability distributions that extend well-known families of distributions and at the same time provide great flexibility in modeling data in practice.

In many practical situations, classical distributions do not provide adequate fits to real data. For example, if the data are asymmetric, the normal distribution will not be a good choice. So, several generators employing one or more parameters to generate new distributions have been proposed in the statistical literature. Some well-known generators are Marshal-Olkin generated family (MO-G) Marshall and Olkin (1997), the beta-G by Eugene, Lee, and Famoye (2002) and Jones (2004), Kumaraswamy-G (Kw-G for short) Cordeiro and de Castro (2011), 
McDonald-G (Mc-G) by Alexander, Cordeiro, Ortega, and Sarabia (2012), gamma-G (type 1) by Zografos and Balakrishnan (2009), gamma-G (type 2) by Ristić and Balakrishnan (2012), gamma-G (type 3) by Torabi and Hedesh (2012), log-gamma-G by Amini, MirMostafaee, and Ahmadi (2012), logistic-G by Tahir, Cordeiro, Alzaatreh, Mansoor, and Zubair (2015a), exponentiated generalized-G by Cordeiro, Ortega, and da Cunha (2013), Transformed-Transformer (T-X) by Alzaatreh, Lee, and Famoye (2013), exponentiated (T-X) by Alzaghal, Famoye, and Lee (2013), Weibull-G by Bourguignon, Silva, and Cordeiro (2014), Exponentiated half logistic generated family by Cordeiro, Alizadeh, and Ortega (2014a), Lomax-G by Cordeiro, Ortega, Popović, and Pescim (2014b), Kumaraswamy Odd log-logistic-G by Alizadeh, Emadi, Doostparast, Cordeiro, Ortega, and Pescim (2015b), Kumaraswamy Marshall-Olkin by Alizadeh, Tahir, Cordeiro, Mansoor, Zubair, and Hamedani (2015c), Beta Marshall-Olkin by Alizadeh, Cordeiro, De Brito, and Demétrio (2015a), Type 1 Half-Logistic family of distributions by Cordeiro, Alizadeh, and Diniz Marinho (2015) and Odd generalized exponential-G by Tahir, Cordeiro, Alizadeh, Mansoor, Zubair, and Hamedani (2015b).

Let $r(t)$ be the probability density function (pdf) of a random variable $T \in[a, b]$ for $-\infty<$ $a<b<\infty$ and let $W[G(x)]$ be a function of the cumulative distribution function (cdf) of a random variable $X$ satisfying the following conditions:

$$
\begin{cases}(i) & W[G(x)] \in[a, b], \\ \text { (ii }) & W[G(x)] \text { is differentiable and monotonically non-decreasing, and } \\ \text { (iii }) & W[G(x)] \rightarrow a \text { as } x \rightarrow-\infty \text { and } W[G(x)] \rightarrow b \text { as } x \rightarrow \infty .\end{cases}
$$

Recently, Alzaatreh et al. (2013) defined the T-X family of distributions by

$$
F(x)=\int_{a}^{W[G(x)]} r(t) d t
$$

where $W[G(x)]$ satisfies the conditions (1). The pdf corresponding to (2) is given by

$$
f(x)=\left\{\frac{d}{d x} W[G(x)]\right\} r\{W[G(x)]\} .
$$

Taking $W[G(x)]=1-(\bar{G}(x))^{\alpha}$ and $r(t)=1+\lambda-2 \lambda t, 0<t<1$, we define the cumulative distribution function (cdf) of the Another Generalized Transmuted Class (AGT-G for short) of distributions by

$$
F(x ; \lambda, \alpha, \boldsymbol{\xi})=(1+\lambda)\left[1-(\bar{G}(x ; \boldsymbol{\xi}))^{\alpha}\right]-\lambda\left[1-(\bar{G}(x ; \boldsymbol{\xi}))^{\alpha}\right]^{2} \quad, \alpha>0,|\lambda| \leq 1
$$

where $G(x ; \boldsymbol{\xi})$ is the baseline cdf depending on a parameter vector $\boldsymbol{\xi}$ and $\alpha>0$ and $|\lambda| \leq 1$ are two additional shape parameters. For each baseline cdf G, the AGT-G family of distributions is defined by the cdf (4). It includes the Transmuted family of distributions and the proportional reversed hazard rate models. Some special models are given in Table 1.

This paper is organized as follows. In Section 2, we define the AGT-G family. Three special cases of this family are defined in Section 3. In Section 4, the asymptotic and shape of the density and hazard rate functions are expressed analytically. Some useful expansions are derived in Section 5. In Section 6, we provide explicit expressions for the moments, incomplete moments, generating function and mean deviation. Extreme values are discussed in Section 7. General expressions for the Rényi and Shannon entropies are presented in Section 8. General results for order statistics are obtained in Section 9. Certain characterisations are given in Section 10. In Section 11, we introduce a bivariate extension of the new family. Estimation procedures of the model parameters are presented in Section 12. Applications to two real data sets illustrate the performance of the new family in Section 13. The paper is concluded in Section 14. 
Table 1: Some known special cases of the AGT-G model.

\begin{tabular}{|c|c|l|l|}
\hline$\alpha$ & $\lambda$ & $G(x)$ & Reduced distribution \\
\hline 1 & - & $G(x)$ & Transmuted G family of distributions Shaw and Buckley (2009) \\
- & 0 & $G(x)$ & Proportioanl hazard rate family Gupta and Gupta (2007) \\
1 & 0 & $G(x)$ & $G(x)$ \\
1 & - & exponentiated exponential & Transmuted exponentiated exponential distribution Merovci (2013a) \\
1 & - & Pareto & Transmuted Pareto distribution Merovci and Puka (2014) \\
1 & - & Gumbel & Transmuted Gumbel distribution Aryal and Tsokos (2009) \\
1 & - & Weibull & Transmuted Weibull distribution Aryal and Tsokos (2011) \\
1 & - & inverse Weibull & Transmuted inverse Weibull distribution Merovci, Elbatal, and Ahmed (2014) \\
1 & - & Lindley & Transmuted Lindley distribution Merovci (2013b) \\
1 & - & Lindley-geometric & Transmuted Lindley-geometric Merovci and Elbatal (2014a) \\
1 & - & Weibull-geometric & Transmuted Weibull-geometric Merovci and Elbatal (2014b) \\
1 & - & Rayligh & Transmuted Rayligh distribution Merovci (2013c) \\
- & - & Generalized Rayligh & Transmuted Generalized Rayligh distribution Merovci (2014) \\
1 & - & extreme value & Transmuted extreme value distribution Aryal and Tsokos (2009) \\
1 & - & log-logistic & Transmuted log-logistic distribution Aryal (2013) \\
\hline
\end{tabular}

\section{The new family}

The corresponding density function to (4) is given by

$$
f(x ; \lambda, \alpha, \boldsymbol{\xi})=\alpha g(x, \boldsymbol{\xi})(\bar{G}(x, \boldsymbol{\xi}))^{\alpha-1}\left\{1+\lambda-2 \lambda\left[1-(\bar{G}(x ; \boldsymbol{\xi}))^{\alpha}\right]\right\}
$$

where $g(x ; \boldsymbol{\xi})$ is the baseline pdf. Equation (5) will be most tractable when the cdf $G(x)$ and the pdf $g(x)$ have simple analytic expressions. Hereafter, a random variable $X$ with density function (5) is denoted by $X \sim \operatorname{AGT}-\mathrm{G}(\alpha, \lambda, \boldsymbol{\xi})$. Further, we can omit (sometimes) the dependence on the vector $\boldsymbol{\xi}$ of the parameters and simply write $G(x)=G(x ; \boldsymbol{\xi})$.

The hazard rate function (hrf) of $X$ becomes

$$
h(x ; \lambda, \alpha, \boldsymbol{\xi})=\frac{\alpha g(x, \boldsymbol{\xi})(\bar{G}(x, \boldsymbol{\xi}))^{\alpha-1}\left\{1+\lambda-2 \lambda\left[1-(\bar{G}(x ; \boldsymbol{\xi}))^{\alpha}\right]\right\}}{1-(1+\lambda)\left[1-(\bar{G}(x ; \boldsymbol{\xi}))^{\alpha}\right]+\lambda\left[1-(\bar{G}(x ; \boldsymbol{\xi}))^{\alpha}\right]^{2}}
$$

To motivate the new family, let $Z_{1}, Z_{2}$ be i.i.d random variables from $1-(\bar{G}(x ; \boldsymbol{\xi}))^{\alpha}$ and $Z_{1: 2}=\min \left\{Z_{1}, Z_{2}\right\}$ and $Z_{2: 2}=\max \left\{Z_{1}, Z_{2}\right\}$, and let

$$
V= \begin{cases}Z_{1: 2}, & \text { with probability } \frac{1+\lambda}{2} ; \\ Z_{2: 2}, & \text { with probability } \frac{1-\lambda}{2} .\end{cases}
$$

Then $F_{V}(x ; \lambda, \alpha, \boldsymbol{\xi})=(1+\lambda)\left[1-(\bar{G}(x ; \boldsymbol{\xi}))^{\alpha}\right]-\lambda\left[1-(\bar{G}(x ; \boldsymbol{\xi}))^{\alpha}\right]^{2}$, which is the proposed family. The AGT-G family of distributions is easily simulated by inverting (4) as follows: if $U$ has a uniform $U(0,1)$ distribution,then

$$
X_{U}=G^{-1}\left\{1-\left[\frac{\lambda-1+\sqrt{(1+\lambda)^{2}-4 \lambda U}}{2 \lambda}\right]^{\frac{1}{\alpha}}\right\} \text { for } \lambda \neq 0
$$

has the density function (5).

\section{Special AGT-G distributions}

In the following sections, we study some mathematical properties of AGT-G distribution since it extends several widely-known distributions in the literature. First, we discuss some special AGT-G distributions. 


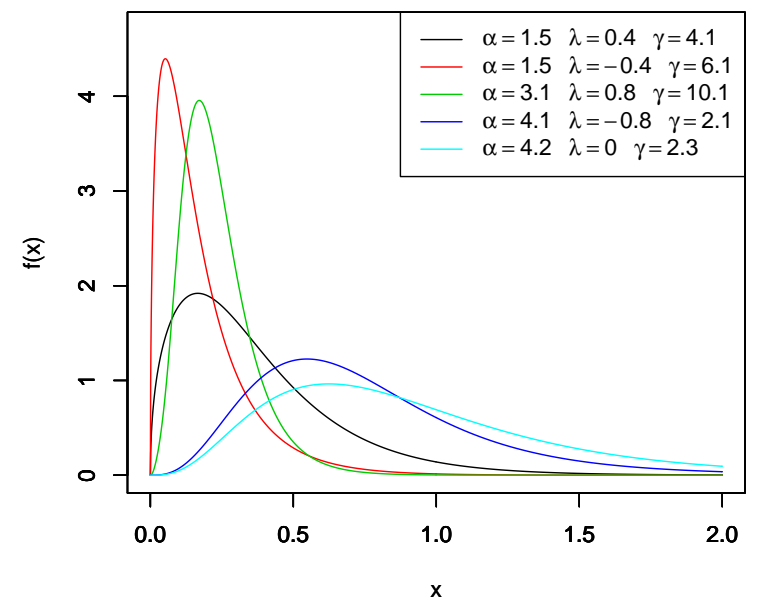

Figure 1: The pdf's of various AGT-E distributions .

\subsection{AGT-Exponential(AGT-E) distribution}

The parent exponential distribution has pdf and cdf given, respectively, by

$$
g(x, \gamma)=\gamma \exp (-\gamma x)
$$

and

$$
G(x, \gamma)=1-\exp (-\gamma x)
$$

The cdf and pdf of AGT-Exponential distribution are given by $(x>0)$

$$
\begin{gathered}
F(x ; \lambda, \alpha, \gamma)=(1+\lambda)[1-\exp (-\alpha \gamma x)]-\lambda[1-\exp (-\alpha \gamma x)]^{2} \quad, \alpha>0,|\lambda| \leq 1 \\
f(x ; \lambda, \alpha, \gamma)=\alpha \gamma \exp (-\alpha \gamma x)\{1+\lambda-2 \lambda[1-\exp (-\alpha \gamma x)]\}
\end{gathered}
$$

Figure 1 illustrates some of the possible shapes of the pdf of the AGT-E distribution. The expectation and variance of AGT-E are:

$$
E(X)=\frac{2-\lambda}{2 \alpha \gamma} \text { and } \operatorname{var}(X)=\frac{4-3 \lambda}{4 \alpha^{2} \gamma^{2}} .
$$

\subsection{AGT-Fréchet (AGT-F) distribution}

The parent Fréchet distribution has cdf and pdf given, respectively, by

$$
G(x ; a, b)=\exp \left(-\left(\frac{b}{x}\right)^{a}\right), \quad a>0, b>0, x>0,
$$

and

$$
g(x ; a, b)=a b^{a} x^{-(a+1)} \exp \left(-\left(\frac{b}{x}\right)^{a}\right)
$$

The cdf and pdf of AGT-Fréchet distribution are given by $(x>0)$ :

$$
\begin{aligned}
F(x ; \lambda, \alpha, a, b) & =(1+\lambda)\left[1-\left[1-\exp \left(-\left(\frac{b}{x}\right)^{a}\right)\right]^{\alpha}\right] \\
& -\lambda\left[1-\left[1-\exp \left(-\left(\frac{b}{x}\right)^{a}\right)\right]^{\alpha}\right]^{2}, \alpha>0,|\lambda| \leq 1, a>0, b>0,
\end{aligned}
$$


and

$$
\begin{gathered}
f(x ; \lambda, \alpha, a, b)=\alpha a b^{a} x^{-(a+1)} \exp \left(-\left(\frac{b}{x}\right)^{a}\right)\left[1-\exp \left(-\left(\frac{b}{x}\right)^{a}\right)\right]^{\alpha-1} \\
\times\left\{1+\lambda-2 \lambda\left[1-\left[1-\exp \left(-\left(\frac{b}{x}\right)^{a}\right)\right]^{\alpha}\right]\right\}
\end{gathered}
$$

\subsection{AGT-Normal(AGT-N) distribution}

The cdf and pdf of AGT-Normal distribution are given by:

$$
\begin{aligned}
F(x ; \lambda, \alpha, \mu, \sigma) & =(1+\lambda)\left[1-\left(1-\Phi\left(\frac{x-\mu}{\sigma}\right)\right)^{\alpha}\right] \\
& -\lambda\left[1-\left(1-\Phi\left(\frac{x-\mu}{\sigma}\right)\right)^{\alpha}\right]^{2}, \alpha>0,|\lambda| \leq 1,
\end{aligned}
$$

and

$$
\begin{aligned}
f(x ; \lambda, \alpha, \mu, \sigma) & =\alpha \phi\left(\frac{x-\mu}{\sigma}\right)\left(1-\Phi\left(\frac{x-\mu}{\sigma}\right)\right)^{\alpha-1} \\
& \times\left\{1+\lambda-2 \lambda\left[1-\left(1-\Phi\left(\frac{x-\mu}{\sigma}\right)\right)^{\alpha}\right]\right\}
\end{aligned}
$$

\subsection{The AGT-Uniform (AGT-U) distribution}

The parent uniform distribution in the interval $(0, \theta), \quad \theta>0$ has cdf and pdf given, respectively, by

$$
G(x ; \theta)=\frac{x}{\theta}
$$

and

$$
g(x ; \theta)=\frac{1}{\theta}
$$

The cdf and pdf of AGT-Uniform distribution are given by:

$$
F(x ; \lambda, \alpha, \theta)=(1+\lambda)\left[1-\left(1-\frac{x}{\theta}\right)^{\alpha}\right]-\lambda\left[1-\left(1-\frac{x}{\theta}\right)^{\alpha}\right]^{2}, \alpha>0,|\lambda| \leq 1
$$

and

$$
f(x ; \lambda, \alpha, \theta)=\frac{\alpha}{\theta}\left(1-\frac{x}{\theta}\right)^{\alpha-1}\left\{1+\lambda-2 \lambda\left[1-\left(1-\frac{x}{\theta}\right)^{\alpha}\right]\right\}
$$

\subsection{The AGT-Weibull (AGT-W) distribution}

The parent Weibull distribution has cdf and pdf given by, respectively:

$$
G(x ; a, b)=1-\exp \left(-b x^{a}\right)
$$

and

$$
g(x ; a, b)=a b x^{a-1} \exp \left(-b x^{a}\right)
$$

The cdf and pdf of AGT-Weibull distribution are given by

$$
\begin{aligned}
F(x ; \lambda, \alpha, a, b) & =(1+\lambda)\left[1-\exp \left(-\alpha b x^{a}\right)\right] \\
& -\lambda\left[1-\exp \left(-\alpha b x^{a}\right)\right]^{2}, \alpha>0,|\lambda| \leq 1
\end{aligned}
$$





Figure 2: The pdf's of various AGT-Weibull distributions .

$$
\begin{aligned}
f(x ; \lambda, \alpha, a, b) & =\alpha a b x^{a-1} \exp \left(-\alpha b x^{a}\right) \\
& \left\{1+\lambda-2 \lambda\left[1-\exp \left(-\alpha b x^{a}\right)\right]\right\}
\end{aligned}
$$

Figure 2 illustrates possible shapes of the density functions for some AGT-Weibull distributions.

\section{Asymptotics and shapes}

Proposition 1 The asymptotics of equations (4), (5) and (6) as $G(x) \rightarrow 0$ are given by

$$
\begin{aligned}
& F(x) \sim \alpha(1+\lambda) G(x) \quad \text { as } G(x) \rightarrow 0, \\
& f(x) \sim \alpha(1+\lambda) g(x) \quad \text { as } G(x) \rightarrow 0, \\
& h(x) \sim \alpha(1+\lambda) g(x) \quad \text { as } G(x) \rightarrow 0 .
\end{aligned}
$$

Proposition 2 The asymptotics of equations (4), (5) and (6) as $x \rightarrow \infty$ are given by

$$
\begin{aligned}
& 1-F(x) \sim(\bar{G}(x))^{\alpha} \quad \text { as } \mathrm{x} \rightarrow \infty, \\
& f(x) \sim \alpha g(x)(\bar{G}(x))^{\alpha-1} \quad \text { as } \mathrm{x} \rightarrow \infty, \\
& h(x) \sim \frac{\alpha g(x)}{\bar{G}(x)} \text { as } \mathrm{x} \rightarrow \infty
\end{aligned}
$$

The shapes of the density and hazard rate functions can be described analytically. The critical points of the AGT-G density function are the roots of the equation

$$
\frac{g^{\prime}(x)}{g(x)}+(1-\alpha) \frac{g(x)}{\bar{G}(x)}-\frac{2 \alpha \lambda g(x)(\bar{G}(x))^{\alpha-1}}{1+\lambda-2 \lambda\left[1-(\bar{G}(x))^{\alpha}\right]}=0 .
$$

The critical points of $h(x)$ are obtained from the equation

$$
\begin{aligned}
& \frac{g^{\prime}(x)}{g(x)}+(1-\alpha) \frac{g(x)}{\bar{G}(x)}-\frac{2 \alpha \lambda g(x)(\bar{G}(x))^{\alpha-1}}{1+\lambda-2 \lambda\left[1-(\bar{G}(x))^{\alpha}\right]} \\
& +\frac{\alpha g(x)(\bar{G}(x))^{\alpha-1}\left\{1+\lambda-2 \lambda\left[1-(\bar{G}(x))^{\alpha}\right]\right\}}{1-(1+\lambda)\left[1-(\bar{G}(x))^{\alpha}\right]+\lambda\left[1-(\bar{G}(x))^{\alpha}\right]^{2}}=0 .
\end{aligned}
$$




\section{Useful expansions}

By using generalized binomial expansion we can show that the cdf (4) of $X$ has the expansion

$$
F(x ; \alpha, \lambda, \boldsymbol{\xi})=\sum_{k=0}^{\infty} c_{k}(G(x))^{k}=\sum_{k=0}^{\infty} c_{k} H_{k}(x)
$$

where $c_{0}=0$ and for $k \geq 1$,

$$
c_{k}=(-1)^{k}\left[(\lambda-1)\left(\begin{array}{l}
\alpha \\
k
\end{array}\right)-\lambda\left(\begin{array}{c}
2 \alpha \\
k
\end{array}\right)\right]
$$

and $H_{a}(x)=(G(x))^{a}$ denotes the exponentiated-G ("exp-G" for short) cumulative distribution. We can prove $\sum_{k=0}^{\infty} c_{k}=1$. Some structural properties of the exp-G distributions are studied by Mudholkar et al. (1996), Gupta and Kundu (2001) and Nadarajah and Kotz (2006), among others.

The density function of $X$ can be expressed as an infinite linear combination of exp-G density functions

$$
f(x ; \alpha, \lambda, \boldsymbol{\xi})=\sum_{k=0}^{\infty} c_{k+1} h_{k+1}(x),
$$

where $h_{k+1}=(k+1) G(x)^{k} g(x)$ ( for $\left.k \geq 0\right)$ is the exp-G density with power parameter $k+1$. Equation (30) reveals that the AGT-G density function is a linear combination of exp-G density functions. Thus, some mathematical properties of the new model can be derived from those properties of the exp-G distribution. For example, the ordinary and incomplete moments and moment generating function (mgf) of $X$ can be obtained from those quantities of the exp-G distribution.

The formulae derived throughout the paper can be easily handled in most symbolic computation software plataforms such as Maple, Mathematica and Matlab. These plataforms have currently the ability to deal with analytic expressions of formidable size and complexity. Established explicit expressions to calculate statistical measures can be more efficient than computing them directly by numerical integration. The infinity limit in these sums can be substituted by a large positive integer such as 20 or 30 for most practical purposes.

\section{Some measures}

\subsection{Moments}

Let $Y_{k}$ be a random variable with exp-G distribution with power parameter $k+1$, i.e., with density $h_{k+1}(x)$. A first formula for the $n$th moment of $X \sim$ AGT-G follows from (30) as

$$
E\left(X^{n}\right)=\sum_{k=0}^{\infty} c_{k+1} E\left(Y_{k}^{n}\right),
$$

where $\sum_{k=0}^{\infty} c_{k}=1$. Expressions for moments of several exp-G distributions are given in Nadarajah and Kotz (2006b), which can be used to obtained $E\left(X^{n}\right)$.

A second formula for $E\left(X^{n}\right)$ can be written from (31) in terms of the G quantile function as

$$
E\left(X^{n}\right)=\sum_{k=0}^{\infty}(k+1) c_{k+1} \tau(n, k),
$$

where $\tau(n, k)=\int_{-\infty}^{\infty} x^{n}(G(x))^{k} g(x) d x=\int_{0}^{1}\left(Q_{G}(u)\right)^{n} u^{k} d u$. Cordeiro, Nadarajah et al. (2011) obtained $\tau(n, k)$ for some well known distribution such as Normal, Beta, Gamma and Weibull distributions, which can be used to find moments of AGT-G. 
For empirical purposes, the shape of many distributions can be usefully described by what we call the incomplete moments. These types of moments play an important role in measuring inequality, for example, income quantiles and Lorenz and Bonferroni curves, which depend on the incomplete moments of a distribution. The $n$th incomplete moment of $X$ is

$$
m_{n}(y)=E\left(X^{n} \mid X<y\right)=\sum_{k=0}^{\infty}(k+1) c_{k+1} \int_{0}^{G(y)}\left(Q_{G}(u)\right)^{n} u^{k} d u .
$$

The last integral can be computed for most $\mathrm{G}$ distributions.

\subsection{Generating function}

Let $M_{X}(t)=E\left(\mathrm{e}^{\mathrm{tX}}\right)$ be mgf of $X \sim \mathrm{AGT}-\mathrm{G}$,then, the first form of $M_{X}(t)$ comes from (30) as

$$
M_{X}(t)=\sum_{k=0}^{\infty} c_{k+1} M_{k}(t),
$$

where $M_{k}(t)$ is the mgf of $Y_{k}$. Hence, $M_{X}(t)$ can be determined from the exp-G generating function.

A second formula for $M_{X}(t)$ can be derived from (30) as

$$
M_{X}(t)=\sum_{i=0}^{\infty}(k+1) c_{k+1} \rho(t, k),
$$

where $\rho(t, k)=\int_{-\infty}^{\infty} \mathrm{e}^{\mathrm{tx}}(\mathrm{G}(\mathrm{x}))^{\mathrm{k}} \mathrm{g}(\mathrm{x}) \mathrm{dx}=\int_{0}^{1} \exp \left[\mathrm{t} \mathrm{Q}_{\mathrm{G}}(\mathrm{u})\right] \mathrm{u}^{\mathrm{k}} \mathrm{du}$.

We can obtain the mgfs of several distributions directly from equation (35).

\subsection{Mean deviation}

The mean deviation about the mean $\left(\delta_{1}=E\left(\left|X-\mu_{1}^{\prime}\right|\right)\right)$ and about the median $\left(\delta_{2}=E(\mid X-\right.$ $M \mid))$ of $X$ can be expressed as

$$
\delta_{1}(X)=2 \mu_{1}^{\prime} F\left(\mu_{1}^{\prime}\right)-2 m_{1}\left(\mu_{1}^{\prime}\right) \quad \text { and } \quad \delta_{2}(X)=\mu_{1}^{\prime}-2 m_{1}(M),
$$

respectively, where $\mu_{1}^{\prime}=E(X), M=\operatorname{Median}(X)$ is the median defined by $M=Q(0.5)$, $F\left(\mu_{1}^{\prime}\right)$ is easily calculated from the cdf (4) and $m_{1}(z)=\int_{-\infty}^{z} x f(x) d x$ is the first incomplete moment obtained from (33) with $n=1$.

Now, we provide two alternative ways to compute $\delta_{1}$ and $\delta_{2}$. A general equation for $m_{1}(z)$ can be derived from (30) as

$$
m_{1}(z)=\sum_{k=0}^{\infty} c_{k+1} J_{k}(z)
$$

where $J_{k}(z)=\int_{-\infty}^{z} x h_{k+1}(x) d x$ is the basic quantity to compute the mean deviation for the exp-G distributions. Hence, the mean deviation in (36) depend only on the mean deviation of the exp-G distribution. So, alternative representations for $\delta_{1}$ and $\delta_{2}$ are

$$
\delta_{1}(X)=2 \mu_{1}^{\prime} F\left(\mu_{1}^{\prime}\right)-2 \sum_{k=0}^{\infty} c_{k+1} J_{k}\left(\mu_{1}^{\prime}\right) \quad \text { and } \quad \delta_{2}(X)=\mu_{1}^{\prime}-2 \sum_{k=0}^{\infty} c_{k+1} J_{k}(M) .
$$

A simple application of $J_{k}(z)$ refers to the the AGT-G distribution discussed in Section 3.5. The exponentiated Weibull with parameter $k+1$ has pdf (for $x>0$ ) given by

$$
h_{k+1}(x)=\frac{(k+1) \eta}{\sigma^{\eta}} x^{\eta-1} \exp \left[-\left(\frac{x}{\sigma}\right)^{\eta}\right]\left\{1-\exp \left[-\left(\frac{x}{\sigma}\right)^{\eta}\right]\right\}^{k}
$$


and then

$$
\begin{aligned}
J_{k}(z) & =\frac{(k+1) \eta}{\sigma^{\eta}} \int_{0}^{z} x^{\eta} \exp \left[-\left(\frac{x}{\sigma}\right)^{\eta}\right]\left\{1-\exp \left[-\left(\frac{x}{\sigma}\right)^{\eta}\right]\right\}^{k} d x \\
& =\frac{(k+1) \eta}{\sigma^{\eta}} \sum_{r=0}^{k}(-1)^{r}\left(\begin{array}{l}
k \\
r
\end{array}\right) \int_{0}^{z} x^{\eta} \exp \left[-(r+1)\left(\frac{x}{\sigma}\right)^{\eta}\right]
\end{aligned}
$$

The last integral is just the incomplete gamma function and then the mean deviation for the AGT-G distribution can be determined from

$$
m_{1}(z)=\sum_{k=0}^{\infty} \sum_{r=0}^{k} \frac{(k+1) b_{k+1}(-1)^{r}\left(\begin{array}{l}
k \\
r
\end{array}\right)}{(r+1)^{1+\eta^{-1}} \sigma^{2 \eta+1}} \gamma\left(1+\eta^{-1},(r+1)\left(\frac{z}{\sigma}\right)^{\eta}\right)
$$

A second general formula for $m_{1}(z)$ can be derived by setting $u=G(x)$ in (30)

$$
m_{1}(z)=\sum_{k=0}^{\infty}(k+1) c_{k+1} T_{k}(z)
$$

where $T_{k}(z)=\int_{0}^{G(z)} Q_{G}(u) u^{k} d u$ is a simple integral defined from the baseline quantile function and $Q_{G}(u)=G^{-1}(u)$.

Remark: Applications of these equations employed to obtain Bonferroni and Lorenz curves defined for a given probability $\pi$ by

$$
B(\pi)=\frac{T(q)}{\pi \mu_{1}^{\prime}} \quad \text { and } \quad L(\pi)=\frac{T(q)}{\mu_{1}^{\prime}},
$$

respectively, where $\mu_{1}^{\prime}=E(X)$ and $q=Q(\pi)$ is the qf of $X$ at $\pi$.

\section{Extreme values}

Let $\bar{X}=\left(X_{1}+\cdots+X_{n}\right) / n$ denote the mean of a random sample from (5), then by the usual central limit theorem $\sqrt{n}(\bar{X}-E(X)) / \sqrt{\operatorname{Var}(X)}$ approaches the standard normal distribution as $n \rightarrow \infty$ under suitable conditions. Sometimes one would be interested in the asymptotics of the extreme values $M_{n}=\max \left\{X_{1}, \ldots, X_{n}\right\}$ and $m_{n}=\min \left\{X_{1}, \ldots, X_{n}\right\}$.

First, suppose $G$ belongs to the max domain of attraction of Gumbel extreme value distribution. Then by Leadbetter, Lindgren, and Rootzén (2012) (chapter 1), there must exist a strictly positive function, say $h(t)$, such that

$$
\lim _{t \rightarrow \infty} \frac{1-G(t+x h(t))}{1-G(t)}=\mathrm{e}^{-x},
$$

for every $x \in(-\infty, \infty)$. But

$$
\lim _{t \rightarrow \infty} \frac{1-F(t+x h(t))}{1-F(t)}=\lim _{x \rightarrow \infty} \frac{x f(t x)}{f(t)}=\mathrm{e}^{-\alpha x},
$$

for every $x \in(-\infty, \infty)$. So, it follows from Leadbetter et al. (2012) (chapter 1) that F belongs to the max domain of attraction of the Gumbel extreme value distribution with

$$
\lim _{n \rightarrow \infty} P\left[a_{n}\left(M_{n}-b_{n} \leq x\right)\right]=\exp [-\exp (-\alpha x)]
$$

for some suitable norming constants $a_{n}>0$ and $b_{n}$. Second, suppose $\mathrm{G}$ belongs to the max domain of attraction of the Fréchet extreme value distribution. Then from Leadbetter et al. (2012) (Chapter 1), there must exist a $\beta>0$ such that

$$
\lim _{t \rightarrow \infty} \frac{1-G(t+x h(t))}{1-G(t)}=x^{c}
$$


for every $x \in(-\infty, \infty)$. But

$$
\begin{aligned}
\lim _{t \rightarrow \infty} \frac{1-F(t+x h(t))}{1-F(t)} & =\lim _{t \rightarrow \infty} \frac{x f(t x)}{f(t)} \\
& =x^{\alpha c}
\end{aligned}
$$

for every $x>0$. So, it follows from Leadbetter et al. (2012) (chapter 1) that F belongs to the max domain of attraction of the Gumbel extreme value distribution with

$$
\lim _{n \rightarrow \infty} P\left[a_{n}\left(M_{n}-b_{n} \leq x\right)\right]=\exp \left(-x^{\alpha c}\right)
$$

for some suitable norming constants $a_{n}>0$ and $b_{n}$. Third, suppose $\mathrm{G}$ belongs to the max domain of attraction of the Weibull extreme value distribution. Then, Leadbetter et al. (2012) (chapter 1 ), there must exist a $\beta>0$ such that

$$
\lim _{t \rightarrow 0} \frac{G(t x)}{G(t)}=x^{\beta}
$$

for every $x<0$. But

$$
\lim _{t \rightarrow 0} \frac{F(t x)}{F(t)}=\lim _{t \rightarrow 0} \frac{x f(t x)}{f(t)}=x^{\beta}
$$

for every $x<0$. So, it follows from Leadbetter et al. (2012) (chapter 1) that F belongs to the max domain of attraction of the Weibull extreme value distribution with

$$
\lim _{n \rightarrow \infty} P\left[a_{n}\left(M_{n}-b_{n} \leq x\right)\right]=\exp \left[-(-x)^{\beta}\right]
$$

for some suitable norming constants $a_{n}>0$ and $b_{n}$. We conclude that $F$ belongs to the same max domain of attraction as that of $\mathrm{G}$. The same argument applies to min domain of attraction. That is, $F$ belongs to the same max domain of attraction as that of $G$.

\section{Entropies}

An entropy is a measure of variation or uncertainty of a random variable $X$. Two popular entropy measures are the Rényi and Shannon entropies Renyi (1961), Shannon (2001). The Rényi entropy of a random variable with pdf $f(x)$ is defined as

$$
I_{R}(\gamma)=\frac{1}{1-\gamma} \log \left(\int_{0}^{\infty} f^{\gamma}(x) d x\right)
$$

for $\gamma>0$ and $\gamma \neq 1$. The Shannon entropy of a random variable $X$ is defined by $E\{-\log [f(X)]\}$. It is the special case of the Rényi entropy when $\gamma \uparrow 1$. Direct calculation yields

$$
\begin{aligned}
\mathrm{E}\{-\log [f(X)]\} & =-\log (\alpha)-\mathrm{E}\{\log [g(X ; \boldsymbol{\xi})]\}+(1-\alpha) \mathrm{E}\{\log [\bar{G}(X ; \boldsymbol{\xi})]\} \\
& -\mathrm{E}\left\{\log \left\{1+\lambda-2 \lambda\left[1-(\bar{G}(X ; \boldsymbol{\xi}))^{\alpha}\right]\right\}\right\}
\end{aligned}
$$

First we define and compute

$$
A\left(a_{1}, a_{2} ; \lambda, \alpha\right)=\int_{0}^{1} x^{a_{1}}\left(1-\frac{2 \lambda}{1+\lambda}\left(1-(1-x)^{\alpha}\right)\right)^{a_{2}} d x .
$$

Using generalized binomial expansion and then after some algebraic manipulations, we obtain

$$
A\left(a_{1}, a_{2} ; \lambda, \alpha\right)=\sum_{i=0}^{\infty} \sum_{j=0}^{i}(-1)^{i+j}\left(\begin{array}{c}
a_{2} \\
i
\end{array}\right)\left(\begin{array}{c}
i \\
j
\end{array}\right)\left(\frac{2 \lambda}{1+\lambda}\right)^{i} \operatorname{Beta}\left(a_{1}+1, \alpha j+1\right)
$$


Proposition 3 Let $X$ be a random variable with $p d f$ (5). Then,

$$
\begin{gathered}
\mathrm{E}\{\log [G(X)]\}=\left.\frac{\alpha}{1+\lambda} \frac{\partial}{\partial t} A(\alpha+t-1,1 ; \lambda, \alpha)\right|_{t=0} \\
\mathrm{E}\left\{\log \left\{1+\lambda-2 \lambda\left[1-\bar{G}(X ; \boldsymbol{\xi})^{\alpha}\right]\right\}\right\}=\left.\frac{\alpha}{1+\lambda} \frac{\partial}{\partial t} \frac{1}{(1+\lambda)^{t}} A(\alpha-1, t+1 ; \lambda, \alpha)\right|_{t=0}
\end{gathered}
$$

The simplest formula for the entropy of $X$ is given by

$$
\begin{aligned}
\mathrm{E}\{-\log [f(X)]\} & =-\log (\alpha)-\mathrm{E}\{\log [g(X ; \boldsymbol{\xi})]\} \\
& +\left.(1-\alpha) \frac{\alpha}{1+\lambda} \frac{\partial}{\partial t} A(\alpha+t-1,1 ; \lambda, \alpha)\right|_{t=0} \\
& -\left.\frac{\alpha}{1+\lambda} \frac{\partial}{\partial t} \frac{1}{(1+\lambda)^{t}} A(\alpha-1, t+1 ; \lambda, \alpha)\right|_{t=0}
\end{aligned}
$$

After some algebraic manipulations, we obtain an alternative expression for $I_{R}(\gamma)$

$$
I_{R}(\gamma)=\frac{\gamma}{1-\gamma} \log \left(\frac{\alpha}{1+\lambda}\right)+\frac{1}{1-\gamma} \log \left\{\sum_{i=0}^{\infty} \sum_{j=0}^{i} w_{i, j}^{*} \mathrm{E}_{Y_{j}}\left[g^{\gamma-1}\left[G^{-1}(Y)\right]\right]\right\}
$$

where $Y_{i} \sim \operatorname{Beta}(\gamma(\alpha-1)+1, \gamma j+1)$ and

$$
w_{i, j}^{*}=\frac{(-1)^{i+j}\left(\begin{array}{l}
\gamma \\
i
\end{array}\right)\left(\begin{array}{l}
i \\
j
\end{array}\right)}{\operatorname{Beta}(\gamma(\alpha-1)+1, \gamma j+1)}\left(\frac{2 \lambda}{1+\lambda}\right)^{i}
$$

\section{Order statistics}

Order statistics make their appearance in many areas of statistical theory and practice. Suppose $X_{1}, \ldots, X_{n}$ is a random sample from the AGT-G family of distributions. We can write the density of the $i$ th order statistic, say $X_{i: n}$, as

$$
f_{i: n}(x)=K f(x)(F(x))^{i-1}\{1-F(x)\}^{n-i}=K \sum_{j=0}^{n-i}(-1)^{j}\left(\begin{array}{c}
n-i \\
j
\end{array}\right) f(x)(F(x))^{j+i-1},
$$

where $K=n ! /[(i-1) !(n-i) !]$.

Following similar algebraic manipulations, we can write the density function of the $i^{t h}$ order statistic, $X_{i: n}$, as

$$
f_{i: n}(x)=\sum_{r, k=0}^{\infty} m_{r, k} h_{r+k+1}(x),
$$

where $h_{r+k+1}(x)$ denotes the exp-G density function with power parameter $r+k+1$,

$$
m_{r, k}=\frac{n !(r+1)(i-1) ! c_{r+1}}{(r+k+1)} \sum_{j=0}^{n-i} \frac{(-1)^{j} f_{j+i-1, k}}{(n-i-j) ! j !}
$$

and $c_{k}$ is defined in equation (29). Here, the quantities $f_{j+i-1, k}$ are obtained recursively by $f_{j+i-1,0}=c_{0}^{j+i-1}$ and (for $\left.k \geq 1\right)$

$$
f_{j+i-1, k}=\left(k c_{0}\right)^{-1} \sum_{m=1}^{k}[m(j+i)-k] c_{m} f_{j+i-1, k-m} .
$$


Equation (41) is the main result of this section. It reveals that the pdf of the AGT-G order statistic is a linear combination of exp-G density functions. So, several mathematical quantities of the AGT-G order statistics such as ordinary, incomplete and factorial moments, mgf, mean deviation and several others can be obtained from those quantities of the exp-G distribution.

\section{Characterization results}

In designing a stochastic model for a particular modeling problem, an investigator will be vitally interested to know if their model fits the requirements of a specific underlying probability distribution. To this end, the investigator will rely on the characterizations of the selected distribution. Generally speaking, the problem of characterizing a distribution is an important problem in various fields and has recently attracted the attention of many researchers. Consequently, various characterization results have been reported in the literature. These characterizations have been established in many different directions. In this Section, we present characterizations of AGT-G distribution. These characterizations are based on: (i) a simple relationship between two truncated moments; (ii) conditional expectations of a function of the random variable. We like to mention that the characterization $(i)$ which is expressed in terms of the ratio of truncated moments is stable in the sense of weak convergence. It also serves as a bridge between a first order differential equation and probability and it does not require a closed form of the cdf.

\subsection{Characterizations based on two truncated moments}

In this subsection we present characterizations of ATG-G distribution in terms of a simple relationship between two truncated moments. Our characterization results presented here will employ an interesting result due to Glänzel (1987) (Theorem 4 below). The advantage of the characterizations given here is that, cdf $F$ need not have a closed form and is given in terms of an integral whose integrand depends on the solution of a first order differential equation, which can serve as a bridge between probability and differential equation.

Theorem 4 Let $(\Omega, \mathcal{F}, \mathbf{P})$ be a given probability space and let $H=[a, b]$ be an interval for some $a<b(a=-\infty, b=\infty$ might as well be allowed $)$. Let $X: \Omega \rightarrow H$ be a continuous random variable with the distribution function $F$ and let $q_{1}$ and $q_{2}$ be two real functions defined on $H$ such that

$$
\mathbf{E}\left[q_{1}(X) \mid X \geq x\right]=\mathbf{E}\left[q_{2}(X) \mid X \geq x\right] \eta(x), \quad x \in H
$$

is defined with some real function $\eta$. Assume that $q_{1}, q_{2} \in C^{1}(H), \eta \in C^{2}(H)$ and $F$ is twice continuously differentiable and strictly monotone function on the set $H$. Finally, assume that the equation $q_{2} \eta=q_{1}$ has no real solution in the interior of $H$. Then $F$ is uniquely determined by the functions $q_{1}, q_{2}$ and $\eta$, particularly

$$
F(x)=\int_{a}^{x} C\left|\frac{\eta^{\prime}(u)}{\eta(u) q_{2}(u)-q_{1}(u)}\right| \exp (-s(u)) d u
$$

where the function $s$ is a solution of the differential equation $s^{\prime}=\frac{\eta^{\prime} q_{2}}{\eta q_{2}-q_{1}} \quad$ and $C$ is a constant, chosen to make $\int_{H} d F=1$. 
Remarks 5 (a) In Theorem 4, the interval $H$ need not be closed since the condition is only on the interior of $H$. (b) Clearly, Theorem 4 can be stated in terms of two functions $q_{1}$ and $\eta$ by taking $q_{2}(x) \equiv 1$, which will reduce the condition given in Theorem 4 to $E\left[q_{1}(X) \mid X \geq x\right]=\eta(x)$. However, adding an extra function will give a lot more flexibility, as far as its application is concerned.

Proposition 6 Let $X: \Omega \rightarrow(0, \infty)$ be a continuous random variable and let $q_{2}(x)=$ $\left\{1-\lambda+2 \lambda(\bar{G}(x))^{\alpha}\right\}^{-1}$ and $q_{1}(x)=q_{2}(x)(\bar{G}(x))$ for $x>0$. The pdf of $X$ is (5) if and only if the function $\eta$ defined in Theorem 4 has the form

$$
\eta(x)=\frac{\alpha}{\alpha+1} \bar{G}(x), \quad x>0 .
$$

Proof. Let $X$ have pdf (5), then

$$
(1-F(x)) \mathbf{E}\left[q_{2}(X) \mid X \geq x\right]=(\bar{G}(x))^{\alpha}, \quad x>0,
$$

and

$$
(1-F(x)) \mathbf{E}\left[q_{1}(X) \mid X \geq x\right]=\frac{\alpha}{\alpha+1}(\bar{G}(x))^{\alpha+1}, \quad x>0,
$$

and finally

$$
\eta(x) q_{2}(x)-q_{1}(x)=-\frac{1}{\alpha+1} q_{2}(x) \bar{G}(x)<0, \quad x>0 .
$$

Conversely, if $\eta$ is given as above, then

$$
s^{\prime}(x)=\frac{\eta^{\prime}(x) q_{2}(x)}{\eta(x) q_{2}(x)-q_{1}(x)}=\alpha g(x)(\bar{G}(x))^{-1}, \quad x>0
$$

and hence

$$
s(x)=-\log \left((\bar{G}(x))^{\alpha}\right), \quad x>0 .
$$

Now, in view of Theorem 4, X has pdf (5).

Corollary 7 Let $X: \Omega \rightarrow(0, \infty)$ be a continuous random variable and let $q_{2}(x)$ be as in Proposition 6. The pdf of $X$ is (5) if and only if there exist functions $q_{1}$ and $\eta$ defined in Theorem 4 satisfying the differential equation

$$
\frac{\eta^{\prime}(x) q_{2}(x)}{\eta(x) q_{2}(x)-q_{1}(x)}=\alpha g(x)(\bar{G}(x))^{-1}, \quad x>0 .
$$

Proof. Is straightforward and hence omitted.

Remarks 8 (a) The general solution of the differential equation in Corollary ?? is

$$
\eta(x)=(\bar{G}(x))^{-\alpha}\left[-\int \alpha g(x)(\bar{G}(x))^{\alpha-1}\left(q_{2}(x)\right)^{-1} q_{1}(x) d x+D\right]
$$


for $x>0$, where $D$ is a constant. One set of appropriate functions is given in Proposition 8 with $D=\frac{1}{2}$.

(b) Clearly there are other triplets of functions $\left(q_{1}, q_{2}, \eta\right)$ satisfying the conditions of Theorem 4. We presented one such triplet in Proposition.

\subsection{Characterizations based on conditional expectation of a function of the variable}

In this subsection we employ a single function $\psi$ of $X$ and state characterization results in terms of $\psi(X)$.

Proposition 9 Let $X: \Omega \rightarrow(a, b)$ be a continuous random variable with cdf $F$. Let $\psi(x)$ be a differentiable function on $(a, b)$ with $\lim _{x \rightarrow a^{+}} \psi(x)=\delta>1$ and $\lim _{x \rightarrow b^{-}} \psi(x)=\infty$. Then

$$
E\left[(\psi(X))^{\delta} \mid X \leq x\right]=\delta(\psi(x))^{\delta-1}, \quad x \in(a, b)
$$

implies

$$
\psi(x)=\delta\left[1-(F(x))^{\frac{1}{\delta-1}}\right]^{-1}, \quad x \in(a, b) .
$$

Proof. From (42), we have

$$
\int_{a}^{x}(\psi(u))^{\delta} f(u) d u=\delta(\psi(x))^{\delta-1} F(x)
$$

Taking derivatives from both sides of the above equation, we arrive at

$$
(\psi(x))^{\delta} f(x)=\delta\left\{(\delta-1) \psi^{\prime}(x)(\psi(x))^{\delta-2} F(x)+(\psi(x))^{\delta-1} f(x)\right\}
$$

from which we have

$$
\frac{f(x)}{F(x)}=(\delta-1)\left\{-\frac{\psi^{\prime}(x)}{\psi(x)}+\frac{\psi^{\prime}(x)}{\psi(x)-\delta}\right\}
$$

Integrating bothe sides of this equation from $x$ to $b$ and using the condition $\lim _{x \rightarrow b^{-}} \psi(x)=$ $\infty$, we obtain (43).

It is easy to see that for $\delta=2$, "implies" in Proposition 8 will be replaced by "if and only if".

Proposition 10 Let $X: \Omega \rightarrow(a, b)$ be a continuous random variable with $c d f$ F. Let $\psi_{1}(x)$ be a differentiable function on $(a, b)$ with $\lim _{x \rightarrow a^{+}} \psi_{1}(x)=\delta / 2>1 / 2$ and $\lim _{x \rightarrow b^{-}} \psi_{1}(x)=$

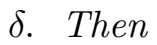

$$
E\left[\left(\psi_{1}(X)\right)^{\delta} \mid X \geq x\right]=\delta\left(\psi_{1}(x)\right)^{\delta-1}, \quad x \in(a, b)
$$

if and only if

$$
\psi_{1}(x)=\delta\left[1+(1-F(x))^{\frac{1}{\delta-1}}\right]^{-1}, \quad x \in(a, b) .
$$


Proof. Is similar to that of Proposition 9.

Remarks 11 (a) Taking, e.g., $(a, b)=(0, \infty)$ and

$$
\psi(x)=\delta\left[1+\left(\left(1-(\bar{G}(x))^{\alpha}\right)\left(1+\lambda(\bar{G}(x))^{\alpha}\right)\right)^{\frac{1}{\delta-1}}\right]^{-1},
$$

Proposition 9 gives a characterization of ATG-G distribution.

(b) Taking, e.g., $(a, b)=(0, \infty)$ and $\psi_{1}(x)=\delta\left[1+\left((\bar{G}(x))^{\alpha}\left(1+\lambda-\lambda(\bar{G}(x))^{\alpha}\right)\right)^{\frac{1}{\delta-1}}\right]^{-1}$, Proposition 10 gives a characterization of $A T G-G$ distribution.

\section{Bivariate extention}

In this section we introduce a bivariate version of the proposed model. The joint cdf is given by

$$
F_{X, Y}(x, y)=(1+\lambda)\left\{1-(1-G(x, y ; \boldsymbol{\xi}))^{\alpha}\right\}-\lambda\left\{1-(1-G(x, y ; \boldsymbol{\xi}))^{\alpha}\right\}^{2}
$$

where $G(x, y ; \boldsymbol{\xi})$ is a bivariate continuous distribution with mariginal cdf's $G_{1}(x ; \boldsymbol{\xi})$ and $G_{2}(y ; \boldsymbol{\xi})$. We denote this distribution by another bivariate Generalized Transmuted $G$ (ABGT$\mathrm{G})$ distribution. The marginal cdf's are given by

$$
\begin{gathered}
F_{X}(x)=(1+\lambda)\left[1-\left(\bar{G}_{1}(x ; \boldsymbol{\xi})\right)^{\alpha}\right]-\lambda\left[1-\left(\bar{G}_{1}(x ; \boldsymbol{\xi})\right)^{\alpha}\right]^{2} \text { and } \\
F_{Y}(y)=(1+\lambda)\left[1-\left(\bar{G}_{2}(y ; \boldsymbol{\xi})\right)^{\alpha}\right]-\lambda\left[1-\left(\bar{G}_{2}(y ; \boldsymbol{\xi})\right)^{\alpha}\right]^{2}
\end{gathered}
$$

The joint pdf of $(X, Y)$ is easily obtained by $f_{X, Y}(x, y)=\frac{\partial^{2} F_{X, Y}(x, y)}{\partial x \partial y}$

$$
f_{X, Y}(x, y)=\alpha A(x, y ; \alpha, \lambda \boldsymbol{\xi})(1-G(x, y ; \boldsymbol{\xi}))^{\alpha-1}\left\{1+\lambda-2 \lambda\left\{1-(1-G(x, y ; \boldsymbol{\xi}))^{\alpha}\right\}\right\}
$$

where

$$
\begin{aligned}
A(x, y ; \alpha, \lambda, \boldsymbol{\xi}) & =g(x, y ; \boldsymbol{\xi})+\frac{1-\alpha}{1-G(x, y ; \boldsymbol{\xi})} \frac{\partial G(x, y, \boldsymbol{\xi})}{\partial x} \frac{\partial G(x, y, \boldsymbol{\xi})}{\partial y} \\
& -\frac{2 \alpha \lambda(1-G(x, y ; \boldsymbol{\xi}))^{\alpha-1}}{1+\lambda-2 \lambda\left\{1-(1-G(x, y ; \boldsymbol{\xi}))^{\alpha}\right\}} \frac{\partial G(x, y, \boldsymbol{\xi})}{\partial x} \frac{\partial G(x, y, \boldsymbol{\xi})}{\partial y}
\end{aligned}
$$

The marginal pdf's are

$$
f_{X}(x)=\alpha g_{1}(x, \boldsymbol{\xi})\left(\bar{G}_{1}(x, \boldsymbol{\xi})\right)^{\alpha-1}\left\{1+\lambda-2 \lambda\left[1-\left(\bar{G}_{1}(x ; \boldsymbol{\xi})\right)^{\alpha}\right]\right\}
$$

and

$$
f_{Y}(y)=\alpha g_{2}(y, \boldsymbol{\xi})\left(\bar{G}_{2}(y, \boldsymbol{\xi})\right)^{\alpha-1}\left\{1+\lambda-2 \lambda\left[1-\left(\bar{G}_{2}(y ; \boldsymbol{\xi})\right)^{\alpha}\right]\right\} .
$$

The conditional cdf's are

$$
F_{X \mid Y}(x \mid y)=\frac{(1+\lambda)\left\{1-(1-G(x, y ; \boldsymbol{\xi}))^{\alpha}\right\}-\lambda\left\{1-(1-G(x, y ; \boldsymbol{\xi}))^{\alpha}\right\}^{2}}{(1+\lambda)\left[1-\left(\bar{G}_{2}(y ; \boldsymbol{\xi})\right)^{\alpha}\right]-\lambda\left[1-\left(\bar{G}_{2}(y ; \boldsymbol{\xi})\right)^{\alpha}\right]^{2}}
$$

and

$$
F_{Y \mid X}(y \mid x)=\frac{(1+\lambda)\left\{1-(1-G(x, y ; \boldsymbol{\xi}))^{\alpha}\right\}-\lambda\left\{1-(1-G(x, y ; \boldsymbol{\xi}))^{\alpha}\right\}^{2}}{(1+\lambda)\left[1-\left(\bar{G}_{1}(x ; \boldsymbol{\xi})\right)^{\alpha}\right]-\lambda\left[1-\left(\bar{G}_{1}(x ; \boldsymbol{\xi})\right)^{\alpha}\right]^{2}}
$$


The conditional density functions are

$$
f_{X \mid Y}(x \mid y)=\frac{A(x, y ; \alpha, \lambda \boldsymbol{\xi})(1-G(x, y ; \boldsymbol{\xi}))^{\alpha-1}\left\{1+\lambda-2 \lambda\left\{1-(1-G(x, y ; \boldsymbol{\xi}))^{\alpha}\right\}\right\}}{g_{2}(y, \boldsymbol{\xi})\left(\bar{G}_{2}(y, \boldsymbol{\xi})\right)^{\alpha-1}\left\{1+\lambda-2 \lambda\left[1-\left(\bar{G}_{2}(y ; \boldsymbol{\xi})\right)^{\alpha}\right]\right\}}
$$

and

$$
f_{Y \mid X}(y \mid x)=\frac{A(x, y ; \alpha, \lambda \boldsymbol{\xi})(1-G(x, y ; \boldsymbol{\xi}))^{\alpha-1}\left\{1+\lambda-2 \lambda\left\{1-(1-G(x, y ; \boldsymbol{\xi}))^{\alpha}\right\}\right\}}{g_{1}(x, \boldsymbol{\xi})\left(\bar{G}_{1}(x, \boldsymbol{\xi})\right)^{\alpha-1}\left\{1+\lambda-2 \lambda\left[1-\left(\bar{G}_{1}(x ; \boldsymbol{\xi})\right)^{\alpha}\right]\right\}}
$$

\section{Estimation}

Here, we determine the maximum likelihood estimates (MLEs) of the model parameters of AGT-G from complete samples only. Let $x_{1}, \ldots, x_{n}$ be observed values from the AGT-G distribution with parameters $\alpha, \lambda$ and $\boldsymbol{\xi}$. Let $\Theta=(\alpha, \lambda, \boldsymbol{\xi})^{\top}$ be the $r \times 1$ parameter vector. The total log-likelihood function for $\Theta$ is given by

$$
\begin{aligned}
\ell_{n} & =\ell_{n}(\Theta)=n \log (\alpha)+\sum_{i=1}^{n} \log \left[g\left(x_{i} ; \boldsymbol{\xi}\right)\right]+(\alpha-1) \sum_{i=1}^{n} \log \left[\bar{G}\left(x_{i} ; \boldsymbol{\xi}\right)\right] \\
& +\sum_{i=1}^{n} \log \left\{1+\lambda-2 \lambda\left[1-\left(\bar{G}\left(x_{i} ; \boldsymbol{\xi}\right)\right)^{\alpha}\right]\right\}
\end{aligned}
$$

The log-likelihood function can be maximized either directly by using the SAS (PROC NLMIXED) or the Ox program (sub-routine MaxBFGS) (see Doornik, 2007) or by solving the nonlinear likelihood equations obtained by differentiating (47). The components of the score function $U_{n}(\Theta)=\left(\partial \ell_{n} / \partial \alpha, \partial \ell_{n} / \partial \lambda, \partial \ell_{n} / \partial \boldsymbol{\xi}\right)^{\top}$ are

$$
\begin{aligned}
\frac{\partial \ell_{n}}{\partial \alpha} & =\frac{n}{\alpha}+\sum_{i=1}^{n} \log \left[\bar{G}\left(x_{i} ; \boldsymbol{\xi}\right)\right]+2 \lambda \sum_{i=1}^{n} \frac{\left(\bar{G}\left(x_{i} ; \boldsymbol{\xi}\right)\right)^{\alpha} \log \left[\bar{G}\left(x_{i} ; \boldsymbol{\xi}\right)\right]}{1+\lambda-2 \lambda\left[1-\left(\bar{G}\left(x_{i} ; \boldsymbol{\xi}\right)\right)^{\alpha}\right]}, \\
\frac{\partial \ell_{n}}{\partial \lambda} & =\sum_{i=1}^{n} \frac{1-2\left[1-\left(\bar{G}\left(x_{i} ; \boldsymbol{\xi}\right)\right)^{\alpha}\right]}{1+\lambda-2 \lambda\left[1-\left(\bar{G}\left(x_{i} ; \boldsymbol{\xi}\right)\right)^{\alpha}\right]}, \\
\frac{\partial \ell_{n}}{\partial \boldsymbol{\xi}} & =\sum_{i=1}^{n} \frac{g^{(\xi)}\left(x_{i}, \boldsymbol{\xi}\right)}{g\left(x_{i}, \boldsymbol{\xi}\right)}+(1-\alpha) \sum_{i=1}^{n} \frac{G^{(\xi)}\left(x_{i}, \boldsymbol{\xi}\right)}{\bar{G}\left(x_{i}, \boldsymbol{\xi}\right)} \\
& +2 \alpha \lambda \sum_{i=1}^{n} \frac{G^{(\xi)}\left(x_{i}, \boldsymbol{\xi}\right)\left(\bar{G}\left(x_{i} ; \boldsymbol{\xi}\right)\right)^{\alpha-1}}{1+\lambda-2 \lambda\left[1-\left(\bar{G}\left(x_{i} ; \boldsymbol{\xi}\right)\right)^{\alpha}\right]}
\end{aligned}
$$

where $h^{(\xi)}(\cdot)$ denotes the derivative of the function $h$ with respect to $\xi$.

\subsection{Maximum product spacing estimates}

The maximum product spacing (MPS) method has been proposed by Cheng and Amin (1983). This method is based on an idea that the differences (spacings) of the consecutive points should be identically distributed. The geometric mean of the differences is given as

$$
G M=\sqrt[n+1]{\prod_{i=1}^{n+1} D_{i}}
$$

where the difference $D_{i}$ is defined by

$$
D_{i}=\int_{x_{(i-1)}}^{x_{(i)}} f(x, \lambda, \alpha, \boldsymbol{\xi}) d x ; \quad i=1,2, \ldots, n+1 .
$$


Here, $F\left(x_{(0)}, \lambda, \alpha, \xi\right)=0$ and $F\left(x_{(n+1)}, \lambda, \alpha, \boldsymbol{\xi}\right)=1$. The MPS estimators $\hat{\lambda}_{P S}, \hat{\alpha}_{P S}$ and $\hat{\boldsymbol{\xi}}_{P S}$ of $\lambda, \alpha$ and $\boldsymbol{\xi}$ are obtained by maximizing the geometric mean (GM) of the differences. Substituting pdf of AGT-G in (49) and taking logarithm of the above expression, we will have

$$
\log G M=\frac{1}{n+1} \sum_{i=1}^{n+1} \log \left[F\left(x_{(i)}, \lambda, \alpha, \boldsymbol{\xi}\right)-F\left(x_{(i-1)}, \lambda, \alpha, \boldsymbol{\xi}\right)\right] .
$$

The MPS estimators $\hat{\lambda}_{P S}, \hat{\alpha}_{P S}$ and $\hat{\boldsymbol{\xi}}_{P S}$ of $\lambda, \alpha$ and $\boldsymbol{\xi}$ can be obtained as the simultaneous solution of the following non-linear equations:

$$
\begin{aligned}
& \frac{\partial \log G M}{\partial \lambda}=\frac{1}{n+1} \sum_{i=1}^{n+1}\left[\frac{F_{\lambda}^{\prime}\left(x_{(i)}, \lambda, \alpha, \boldsymbol{\xi}\right)-F_{\lambda}^{\prime}\left(x_{(i-1)}, \lambda, \alpha, \boldsymbol{\xi}\right)}{F\left(x_{(i)}, \lambda, \alpha, \boldsymbol{\xi}\right)-F\left(x_{(i-1)}, \lambda, \alpha, \boldsymbol{\xi}\right)}\right]=0 \\
& \frac{\partial \log G M}{\partial \alpha}=\frac{1}{n+1} \sum_{i=1}^{n+1}\left[\frac{F_{\alpha}^{\prime}\left(x_{(i)}, \lambda, \alpha, \boldsymbol{\xi}\right)-F_{\alpha}^{\prime}\left(x_{(i-1)}, \lambda, \alpha, \boldsymbol{\xi}\right)}{F\left(x_{(i)}, \lambda, \alpha, \boldsymbol{\xi}\right)-F\left(x_{(i-1)}, \lambda, \alpha, \boldsymbol{\xi}\right)}\right]=0 \\
& \frac{\partial \log G M}{\partial \boldsymbol{\xi}}=\frac{1}{n+1} \sum_{i=1}^{n+1}\left[\frac{F_{\boldsymbol{\xi}}^{\prime}\left(x_{(i)}, \lambda, \alpha, \boldsymbol{\xi}\right)-F_{\boldsymbol{\xi}}^{\prime}\left(x_{(i-1)}, \lambda, \alpha, \boldsymbol{\xi}\right)}{F\left(x_{(i)}, \lambda, \alpha, \boldsymbol{\xi}\right)-F\left(x_{(i-1)}, \lambda, \alpha, \boldsymbol{\xi}\right)}\right]=0
\end{aligned}
$$

\subsection{Least square estimates}

Let $x_{1: n}, x_{2: n}, \ldots, x_{n: n}$ be the ordered sample of size $n$ drawn the AGT-G population pdf. Then, the expectation of the empirical cumulative distribution function is defined as

$$
E\left[F\left(X_{i: n}\right)\right]=\frac{i}{n+1} ; i=1,2, \ldots, n
$$

The least square estimates (LSEs) $\hat{\lambda}_{L S}, \hat{\alpha}_{L S}$ and $\hat{\boldsymbol{\xi}}_{L S}$ of $\lambda, \alpha$ and $\boldsymbol{\xi}$ are obtained by minimizing

$$
Z(\lambda, \alpha, \boldsymbol{\xi})=\sum_{i=1}^{n}\left(F\left(x_{i: n}, \lambda, \alpha, \boldsymbol{\xi}\right)-\frac{i}{n+1}\right)^{2}
$$

Therefore, $\hat{\lambda}_{L S}, \hat{\alpha}_{L S}$ and $\hat{\boldsymbol{\xi}}_{L S}$ of $\lambda, \alpha$ and $\boldsymbol{\xi}$ can be obtained as the solution of the following system of equations:

$$
\begin{gathered}
\frac{\partial Z(\lambda, \alpha, \boldsymbol{\xi})}{\partial \lambda}=\sum_{i=1}^{n} F_{\lambda}^{\prime}\left(x_{i: n}, \lambda, \alpha, \boldsymbol{\xi}\right)\left(F\left(x_{i: n}, \lambda, \alpha, \boldsymbol{\xi}\right)-\frac{i}{n+1}\right)=0 \\
\frac{\partial Z(\lambda, \alpha, \boldsymbol{\xi})}{\partial \alpha}=\sum_{i=1}^{n} F_{\alpha}^{\prime}\left(x_{i: n}, \lambda, \alpha, \boldsymbol{\xi}\right)\left(F\left(x_{i: n}, \lambda, \alpha, \boldsymbol{\xi}\right)-\frac{i}{n+1}\right)=0 \\
\frac{\partial Z(\lambda, \alpha, \boldsymbol{\xi})}{\partial \boldsymbol{\xi}}=\sum_{i=1}^{n} F_{\boldsymbol{\xi}}^{\prime}\left(x_{i: n}, \lambda, \alpha, \boldsymbol{\xi}\right)\left(F\left(x_{i: n}, \alpha, \theta\right)-\frac{i}{n+1}\right)=0
\end{gathered}
$$

\section{Applications}

Now we use a real data set to show that the AGT-E can be a better model than the beta-exponential (Nadarajah and Kotz (2006a)), Kumaraswamy-exponential distribution and exponential distribtuion.

We consider a data set of the life of fatigue fracture of Kevlar 373/epoxy that are subject to constant pressure at the $90 \%$ stress level until all had failed, so we have complete data with the exact times of failure. For previous studies with the data sets see Andrews \& Herzberg (1985) and Barlow, Toland \& Freeman (1984).

These data are:

$0.0251,0.0886,0.0891,0.2501,0.3113,0.3451,0.4763,0.5650,0.5671,0.6566,0.6748,0.6751,0.6753$, $0.7696,0.8375,0.8391,0.8425,0.8645,0.8851,0.9113,0.9120,0.9836,1.0483,1.0596,1.0773,1.1733$, $1.2570,1.2766,1.2985,1.3211,1.3503,1.3551,1.4595,1.4880,1.5728,1.5733,1.7083,1.7263,1.7460$, 
Table 2: Estimated parameters of the AGT-E, BE and KwE distribution for data set.

\begin{tabular}{llllll}
\hline Model & ML Estimate & Standard Error & $-\ell(\cdot ; x)$ & LSE & PS Estimator \\
\hline AGTE & $\hat{\lambda}=0.733$ & 0.274 & 121.3219 & -0.636 & -0.760 \\
& $\hat{\alpha}=1.197$ & 0.344 & & 1.631 & 1.038 \\
& $\hat{\gamma}=0.769$ & 0.101 & & 0.907 & 0.704 \\
\hline Exponential & $\hat{\lambda}=0.510$ & 0.058 & 127.114 & 0.981 & 0.926 \\
\hline Beta & $\hat{a}=1.679$ & 0.374 & 122.227 & 2.235 & 1.520 \\
Exponential & $\hat{b}=1.508$ & 6.760 & & 1.558 & 1.082 \\
& $\hat{\lambda}=0.484$ & 1.981 & & 0.586 & 0.598 \\
\hline Kumaraswamy & $\hat{a}=1.556$ & 0.401 & 122.0942 & 1.987 & 1.426 \\
Exponential & $\hat{b}=2.448$ & 6.065 & & 2.228 & 2.243 \\
& $\hat{\lambda}=0.328$ & 0.691 & & 0.453 & 0.316 \\
& & & & & \\
\hline
\end{tabular}

$1.7630,1.7746,1.8275,1.8375,1.8503,1.8808,1.8878,1.8881,1.9316,1.9558,2.0048,2.0408,2.0903$, $2.1093,2.1330,2.2100,2.2460,2.2878,2.3203,2.3470,2.3513,2.4951,2.5260,2.9911,3.0256,3.2678$, $3.4045,3.4846,3.7433,3.7455,3.9143,4.8073,5.4005,5.4435,5.5295,6.5541,9.0960$

The variance covariance matrix $I(\hat{\theta})^{-1}$ of the MLEs under the AGT-E distribution is computed as

$$
\left(\begin{array}{ccc}
0.075308090 & -0.07199734 & -0.005229209 \\
-0.071997344 & 0.11877571 & 0.021537278 \\
-0.005229209 & 0.02153728 & 0.010247905
\end{array}\right) .
$$

Thus, the variances of the MLE of $\lambda, \alpha$ and $\gamma$ is $\operatorname{var}(\hat{\lambda})=0.075472979, \operatorname{var}(\hat{\alpha})=0.11889578$ and $\operatorname{var}(\hat{\gamma})=0.010248121$.

The LR test statistic to test the hypotheses $H_{0}: \lambda=0 \& \alpha=1$ versus $H_{1}: \lambda \neq 0 \& \alpha \neq 1$ for data set is $\omega=11.584>5.991=\chi_{2 ; 0.05}^{2}$, so we reject the null hypothesis.

Table 3: Criteria for comparison.

\begin{tabular}{llllll}
\hline Model & K-S & $-2 \ell$ & AIC & AICC & BIC \\
\hline AGTE & 0.0954 & 242.643 & 248.643 & 249.143 & 255.636 \\
Beta-E & 0.0962 & 244.455 & 250.455 & 250.621 & 257.447 \\
Kw-E & 0.0988 & 244.188 & 250.188 & 250.521 & 257.180 \\
Expoential & 0.512 & 254.228 & 256.228 & 256.282 & 258.559 \\
\hline
\end{tabular}

In order to compare the two distribution models, we consider criteria like Kolmogorov-Smirnov (K-S) statistics, $-2 \ell$, AIC (Akaike information criterion), and AICC (corrected Akaike information criterion) for the data set. The better distribution corresponds to smaller KS, $-2 \ell$, AIC,AICC and BIC values:

- K-S distance $D_{n}=\sup _{x}\left|F(x)-F_{n}(x)\right|$, where, $F_{n}(x)$ is the empirical distribution function,

- $A I C=-2 \log \ell(\underset{\sim}{x}, \alpha, \lambda, \boldsymbol{\xi})+2 p$,

- $A I C C=A I C+\frac{2 p(p+1)}{n-p-1}$,

- $B I C=-2 \log \ell(\underset{\sim}{x}, \alpha, \lambda, \boldsymbol{\xi})+p \log (n)$, where, $\mathrm{p}$ is the number of parameters are to be estimated from the data and $n$ the sample size.

Also, here for calculating the values of KS we use the sample estimates of $\alpha, \lambda$ and $\gamma$. Table 3 shows the MLEs under both distributions, Table 3 shows the values of KS, $-2 \ell$, AIC, AICC, and BIC values. The values in Table 3 indicate that the AGT-E leads to a better fit than the exponential distribution. The P-P plots, fitted distribution function and density functions of the considered models are plotted in Figures 3 and 4, respectively, for the data set. 

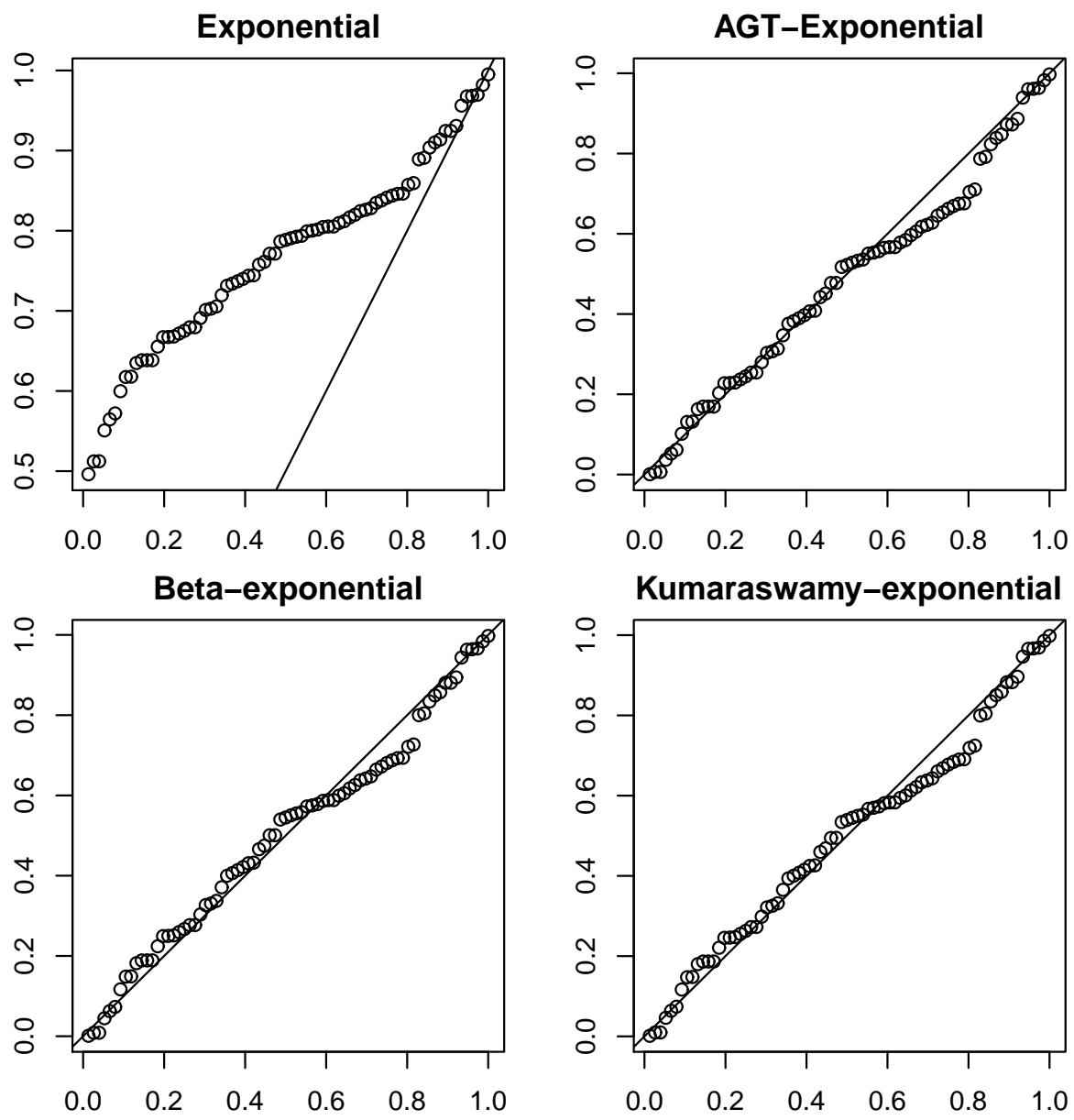

Figure 3: The P-P plots for the real data set 


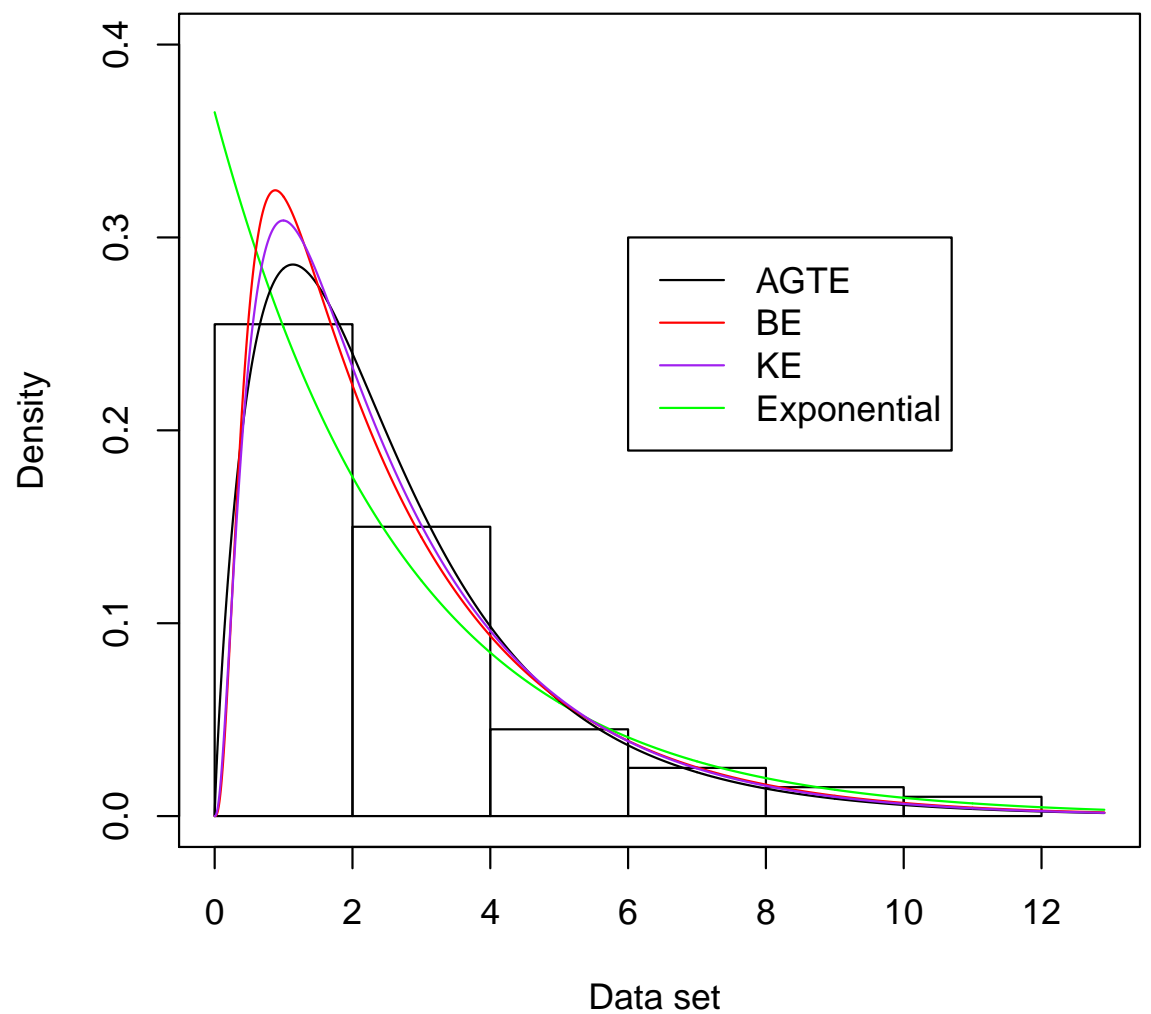

Figure 4: Fitted pdfs plots of the considered distributions for the real data set 


\section{References}

Alexander C, Cordeiro GM, Ortega EM, Sarabia JM (2012). "Generalized Beta-generated Distributions." Computational Statistics \& Data Analysis, 56(6), 1880-1897.

Alizadeh M, Cordeiro GM, De Brito E, Demétrio CGB (2015a). "The Beta Marshall-Olkin Family of Distributions." Journal of Statistical Distributions and Applications, 2(1), 1-18.

Alizadeh M, Emadi M, Doostparast M, Cordeiro GM, Ortega EM, Pescim RR (2015b). "A New Family of Distributions: the Kumaraswamy Odd Log-logistic, Properties and Applications." Hacettepa Journal of Mathematics and Statistics (to appear).

Alizadeh M, Tahir M, Cordeiro GM, Mansoor M, Zubair M, Hamedani G (2015c). "The Kumaraswamy Marshal-Olkin Family of Distributions." Journal of the Egyptian Mathematical Society.

Alzaatreh A, Lee C, Famoye F (2013). "A New Method for Generating Families of Continuous Distributions." Metron, 71(1), 63-79.

Alzaghal A, Famoye F, Lee C (2013). "Exponentiated T-X Family of Distributions with Some Applications." International Journal of Statistics and Probability, 2(3), p31.

Amini M, MirMostafaee S, Ahmadi J (2012). "Log-gamma-generated Families of Distributions." Statistics, (ahead-of-print), 1-20.

Aryal GR (2013). "Transmuted Log-logistic Distribution." Journal of Statistics Applications E Probability, 2(1), 11-20.

Aryal GR, Tsokos CP (2009). "On the Transmuted Extreme Value Distribution with Application." Nonlinear Analysis: Theory, Methods \& Applications, 71(12), e1401-e1407.

Aryal GR, Tsokos CP (2011). "Transmuted Weibull Distribution: A Generalization of the Weibull Probability Distribution." European Journal of Pure and Applied Mathematics, 4(2), 89-102.

Bourguignon M, Silva RB, Cordeiro GM (2014). "The Weibull-G Family of Probability Distributions." Journal of Data Science, 12(1), 53-68.

Cheng R, Amin N (1983). "Estimating Parameters in Continuous Univariate Distributions with a Shifted Origin." Journal of the Royal Statistical Society. Series B (Methodological), pp. 394-403.

Cordeiro GM, Alizadeh M, Diniz Marinho PR (2015). "The Type I Half-logistic Family of Distributions." Journal of Statistical Computation and Simulation, (ahead-of-print), 1-22.

Cordeiro GM, Alizadeh M, Ortega EM (2014a). "The Exponentiated Half-Logistic Family of Distributions: Properties and Applications." Journal of Probability and Statistics, 2014.

Cordeiro GM, de Castro M (2011). "A New Family of Generalized Distributions." Journal of Statistical Computation and Simulation, 81(7), 883-898.

Cordeiro GM, Nadarajah S, et al. (2011). "Closed-form Expressions for Moments of a Class of Beta Generalized Distributions." Brazilian journal of probability and statistics, 25(1), 14-33.

Cordeiro GM, Ortega EM, da Cunha DC (2013). "The Exponentiated Generalized Class of Distributions." Journal of Data Science, 11(1), 1-27.

Cordeiro GM, Ortega EM, Popović BV, Pescim RR (2014b). "The Lomax Generator of Distributions: Properties, Minification Process and Regression Model." Applied Mathematics and Computation, 247, 465-486.

Eugene N, Lee C, Famoye F (2002). "Beta-normal Distribution and Its Applications." Communications in Statistics-Theory and methods, 31(4), 497-512.

Glänzel W (1987). "A Characterization Theorem Based on Truncated Moments and Its Application to Some Distribution Families." In Mathematical statistics and probability theory, pp. 75-84. Springer.

Gupta RC, Gupta RD (2007). "Proportional Reversed Hazard Rate Model and Its Applications." Journal of Statistical Planning and Inference, 137(11), 3525-3536. 
Jones M (2004). "Families of Distributions Arising from Distributions of Order Statistics." Test, 13(1), $1-43$.

Leadbetter MR, Lindgren G, Rootzén H (2012). Extremes and Related Properties of Random Sequences and Processes. Springer Science \& Business Media.

Marshall AW, Olkin I (1997). "A New Method for Adding a Parameter to a Family of Distributions with Application to the Exponential and Weibull Families." Biometrika, 84(3), 641-652.

Merovci F (2013a). "Transmuted Exponentiated Exponential Distribution." Mathematical Sciences And Applications E-Notes, 1(2), 112-122.

Merovci F (2013b). "Transmuted Lindley Distribution." International Journal of Open Problems in Computer Science \& Mathematics, 6(2), 63-72.

Merovci F (2013c). "Transmuted Rayleigh Distribution." Austrian Journal of Statistics, 42(1), 21-31.

Merovci F (2014). "Transmuted Generalized Rayleigh Distribution." Journal of Statistics Applications and Probability, 3(1), 9-20.

Merovci F, Elbatal I (2014a). "Transmuted Lindley-geometric Distribution and Its Applications." Journal of Statistics Applications \& Probability, 3(1), 77-91.

Merovci F, Elbatal I (2014b). "Transmuted Weibull-geometric Distribution and Its Applications." School of Mathematics Northwest University Xian, Shaanxi, PR China, 10(1), 68-82.

Merovci F, Elbatal I, Ahmed A (2014). "The Transmuted Generalized Inverse Weibull Distribution." Austrian Journal of Statistics, 43(2), 119-131.

Merovci F, Puka L (2014). "Transmuted Pareto Distribution." Probstat, 7, 1-11.

Nadarajah S, Kotz S (2006a). "The Beta Exponential Distribution." Reliability engineering \& system safety, 91(6), 689-697.

Nadarajah S, Kotz S (2006b). "The Exponentiated Type Distributions." Acta Applicandae Mathematica, 92(2), 97-111.

Renyi A (1961). "On Measures of Entropy and Information." In Proceedings of the Fourth Berkeley Symposium on Mathematical Statistics and Probability, Volume 1: Contributions to the Theory of Statistics, pp. 547-561. University of California Press, Berkeley, Calif. URL http://projecteuclid.org/ euclid.bsmsp/1200512181.

Ristić MM, Balakrishnan N (2012). "The Gamma-exponentiated Exponential Distribution." Journal of Statistical Computation and Simulation, 82(8), 1191-1206.

Shannon CE (2001). "A mathematical theory of communication." ACM SIGMOBILE Mobile Computing and Communications Review, 5(1), 3-55.

Shaw WT, Buckley IR (2009). "The Alchemy of Probability Distributions: beyond Gram-Charlier Expansions, and a Skew-kurtotic-normal Distribution from a Rank Transmutation Map." arXiv preprint arXiv:0901.0434.

Tahir M, Cordeiro GM, Alzaatreh A, Mansoor M, Zubair M (2015a). "The Logistic-X Family of Distributions and Its Applications." Commun. Stat. Theory Methods (2015a). forthcoming.

Tahir MH, Cordeiro GM, Alizadeh M, Mansoor M, Zubair M, Hamedani GG (2015b). "The Odd Generalized Exponential Family of Distributions with Applications." Journal of Statistical Distributions and Applications, 2(1), 1-28.

Torabi H, Hedesh NM (2012). "The Gamma-uniform Distribution and Its Applications." Kybernetika, (1), 16-30.

Zografos K, Balakrishnan N (2009). "On Families of Beta-and Generalized Gamma-generated Distributions and Associated Inference." Statistical Methodology, 6(4), 344-362. 


\author{
Affiliation: \\ Faton Merovci \\ University of Mitrovica "Isa Boletini" \\ PIM Trepça 40000, Mitrovicë, Kosovo \\ E-mail: fmerovci@yahoo.com \\ Morad Alizadeh \\ Department of Statistics, Faculty of Sciences, \\ Persian Gulf University, Bushehr, 75169, Iran. \\ E-mail: moradalizadeh78@gmail.com \\ G. G. Hamedani \\ Department of Mathematics, Statistics and Computer Science, \\ Marquette University, USA. \\ E-mail: gholamhoss.hamedani@marquette.edu
}

\section{Austrian Journal of Statistics}

published by the Austrian Society of Statistics

Volume 45

September 2016 http://www.ajs.or.at/

http://www.osg.or.at/

Submitted: 2015-05-08

Accepted: 2015-09-14 